\section{Mechanotransduction through growth-factor shedding into the extracellular space}

Daniel J. Tschumperlin ${ }^{1}$, Guohao Dai ${ }^{3}$, Ivan V. Maly ${ }^{3}$, Tadashi Kikuchi ${ }^{2}$, Lily H. Laiho ${ }^{3}$, Anna K. McVittie ${ }^{3}$, Kathleen J. Haley ${ }^{2}$, Graig M. Lilly ${ }^{2}$, Peter T. C. So ${ }^{3}$, Douglas A. Lauffenburger ${ }^{3}$, Roger D. Kamm ${ }^{3}$ \& Jeffrey M. Drazen ${ }^{1}$

${ }^{1}$ Physiology Program, Department of Environmental Health, Harvard School of Public Health, and ${ }^{2}$ Pulmonary and Critical Care Division, Department of Medicine, Brigham and Women's Hospital, Harvard Medical School, Boston, Massachusetts 02115, USA

${ }^{3}$ Biological Engineering Division, Massachusetts Institute of Technology, Cambridge, Massachusetts 02139, USA

Physical forces elicit biochemical signalling in a diverse array of cells, tissues and organisms ${ }^{1-3}$, helping to govern fundamental biological processes. Several hypotheses have been advanced that link physical forces to intracellular signalling pathways, but in many cases the molecular mechanisms of mechanotransduction remain elusive ${ }^{1-9}$. Here we find that compressive stress shrinks the lateral intercellular space surrounding epithelial cells, and triggers cellular signalling via autocrine binding of epidermal growth factor family ligands to the epidermal growth factor receptor. Mathematical analysis predicts that constant rate shedding of autocrine ligands into a collapsing lateral intercellular space leads to increased local ligand concentrations that are sufficient to account for the observed receptor signalling; direct experimental comparison of signalling stimulated by compressive stress versus exogenous soluble ligand supports this prediction. These findings establish a mechanism by which mechanotransduction arises from an autocrine ligand-receptor circuit operating in a dynamically regulated extracellular volume, not requiring induction of force-dependent biochemical processes within the cell or cell membrane.

Epithelial and endothelial cells line the surfaces of the body and frequently encounter physical stresses as pressures equilibrate across these cellular barriers ${ }^{10-13}$. We studied the morphological and biochemical responses of normal human bronchial epithelial (NHBE) cells to compressive stress: in vivo they experience such stress as a consequence of airway constriction ${ }^{14}$, and in vitro compressive stress triggers an integrated pro-fibrotic response reminiscent of that seen in chronic asthma ${ }^{15,16}$.

We used two-photon microscopy to visualize the morphology of living NHBE cells both before and during the application of physical stress. Cell-permeant cytoplasmic dyes (Celltracker and calcein AM) were used to visualize the cell bodies, and a cell-impermeant fluorophore-conjugated dextran was used to visualize the extracellular space directly. The cells, which were grown at the air-liquid interface to produce a pseudo-stratified, mucociliary epithelium, were joined by tight junctions at their apical surface, and separated along their basolateral surface by a lateral intercellular space (LIS) comprising $15 \pm 3 \%$ (mean \pm s.d., $n=5$ ) of the total tissue volume. This morphology, including the LIS, is typical of stratified epithelium ${ }^{17}$ and is representative of the airways in $v i v o^{18,19}$.

To expose the cells to a compressive stress similar to that resulting from airway constriction we applied an apical to basal transcellular pressure gradient across the epithelium ${ }^{14,16}$. This compressive stress decreased the thickness of NHBE cell layers an average of $10.6 \pm 1.9 \%$ (mean \pm s.d., $n=7$, Supplementary Table 1$)$. However, total cell volume was not significantly altered by the compressive stress (decrease of $1.6 \pm 2.3 \%, n=7$, Supplementary Table 1 ), suggesting that changes in cell layer height were a result of fluid leaving the LIS. This was confirmed qualitatively by visualizing the extracellular space (Fig. 1). Quantitative determination of LIS volume in tissue specimens before and after exposure to compressive stress demonstrated an $87.3 \pm 10.0 \%$ reduction in LIS volume (mean \pm s.d., $n=5$, Supplementary Table 2). Our findings indicate that although epithelial cell volumes are maintained relatively constant under compressive stress, the LIS is highly compliant and susceptible to large percentage volume changes.

Collapse of the LIS under compressive stress brings the lateral surfaces of adjacent cells closer to one another. The basolateral surfaces of NHBE cells exhibit strong staining for the ErbB1 epidermal growth factor receptor (EGFR, Fig. 2a), in agreement

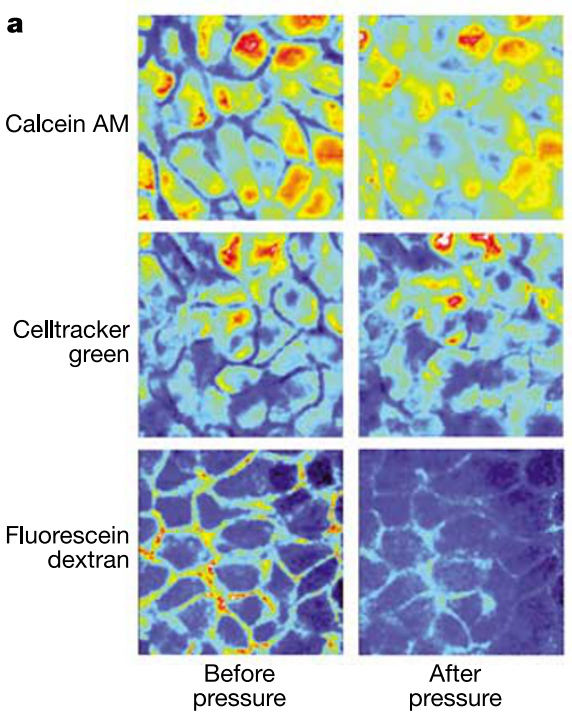

Figure 1 Compliance of the lateral intercellular space. a, Pseudo-colour $x-y$ slices from identical mid-cell layers obtained by two-photon microscopy before and during application of $20 \mathrm{~cm} \mathrm{H} \mathrm{H}_{2} \mathrm{O}$ transcellular pressure. b, A typical pseudo-colour threedimensional reconstruction of bronchial epithelial cells negatively stained with cellimpermeant fluorescein-dextran. Red corresponds to dextran, whereas cooler colours (blue-green) correspond to the absence of dextran. The intensity of dextran staining is plotted along the $z$ axis. The red contour indicates the profile of dextran staining under control conditions, whereas the green profile was obtained in the same tissue 30 min after the onset of a continuous $20 \mathrm{~cm} \mathrm{H}_{2} \mathrm{O}$ transcellular pressure. Note the decrease in cell layer height and loss of LIS volume. 
with observations made in intact human airways and primary airway epithelial cells $\mathrm{s}^{20,21}$. As previously shown ${ }^{22}$, application of compressive stress for $30 \mathrm{~min}$ elicited a robust increase in phosphorylation of the mitogen-activated protein kinase ERK (Fig. 2b). This response was preceded by an increase in phosphorylation of the EGFR at 5-20 min (Fig. 2a). Blockade of the tyrosine kinase domain of the EGFR with basolaterally applied tyrphostin AG 1478 strongly attenuated the mechanical stress-induced phosphorylation of ERK, whereas incubation with tyrphostin AG 1296, which inhibits the tyrosine kinase domain of the platelet-derived growth factor receptor, had no effect on the mechanically induced signal (Fig. 2b). Basolateral application of a monoclonal function-blocking EGFR antibody before compressive stress attenuated the compressive stress-induced ERK phosphorylation in a concentration-dependent manner (Fig. 2b), confirming a role for the extracellular ligandbinding domain of the EGFR. To identify the EGF family member responsible, we applied compressive stress in the presence of increasing basolateral amounts of neutralizing antibodies to EGF, transforming growth factor- $\alpha$ (TGF- $\alpha$ ) and heparin-binding epidermal growth factor (HB-EGF). Whereas the EGF and TGF- $\alpha$ antibodies were ineffective at the highest concentration used, the antibody to HB-EGF attenuated the compressive stress-induced ERK phosphorylation in a dose-dependent manner (Fig. 2b). Incubation with GM 6001, a broad-spectrum inhibitor of matrix metalloprotease activity, was used to inhibit ectodomain shedding of transmembrane EGF family precursors from the basolateral cell surface $^{23,24}$. Its presence attenuated both baseline and transcellular pressure-induced increases in ERK phosphorylation, demonstrat- ing that constitutive sheddase activity for EGF family ligands is present in the LIS, and is required for transduction of the applied mechanical stress. These results demonstrate that compressive stress elicits ERK phosphorylation through a LIS-restricted autocrine pathway involving metalloprotease-dependent shedding of HB-EGF, leading to subsequent binding and activation of the EGFR.

To confirm the relevance of this signalling response in the native setting of the airway wall, we fixed isolated mouse lungs after administration of various bronchially active substances, and examined the distribution of epithelial staining for the active (phosphorylated) form of the EGFR. A 5 min tracheal perfusion with methacholine (MCh; $1 \mathrm{mM}$ ), which was sufficient to maximally constrict the airways, significantly increased the number of airways positive for the phosphorylated EGFR (Fig. 2c). This response to $\mathrm{MCh}$ was specific to the mechanical stress evoked by muscle constriction, as pre-treatment with isoproterenol (Isp), which greatly attenuated constriction, abrogated the EGFR response to MCh.

The localization of the EGFR autocrine loop at the perimeter of the collapsing LIS suggests a proximal role for this receptor system in the transduction of mechanical stress in epithelial cells. We considered the possibility that transient shear stresses generated as fluid is squeezed out of the collapsing LIS might trigger EGFR activation; however, when we applied transcellular pressure gradually as a ramp function over $30 \mathrm{~min}$ to minimize intercellular shear stress, signal transduction was stimulated as effectively as by a step increase in transcellular pressure (Supplementary Fig. 1). We also considered the possibility that the compressive stress response
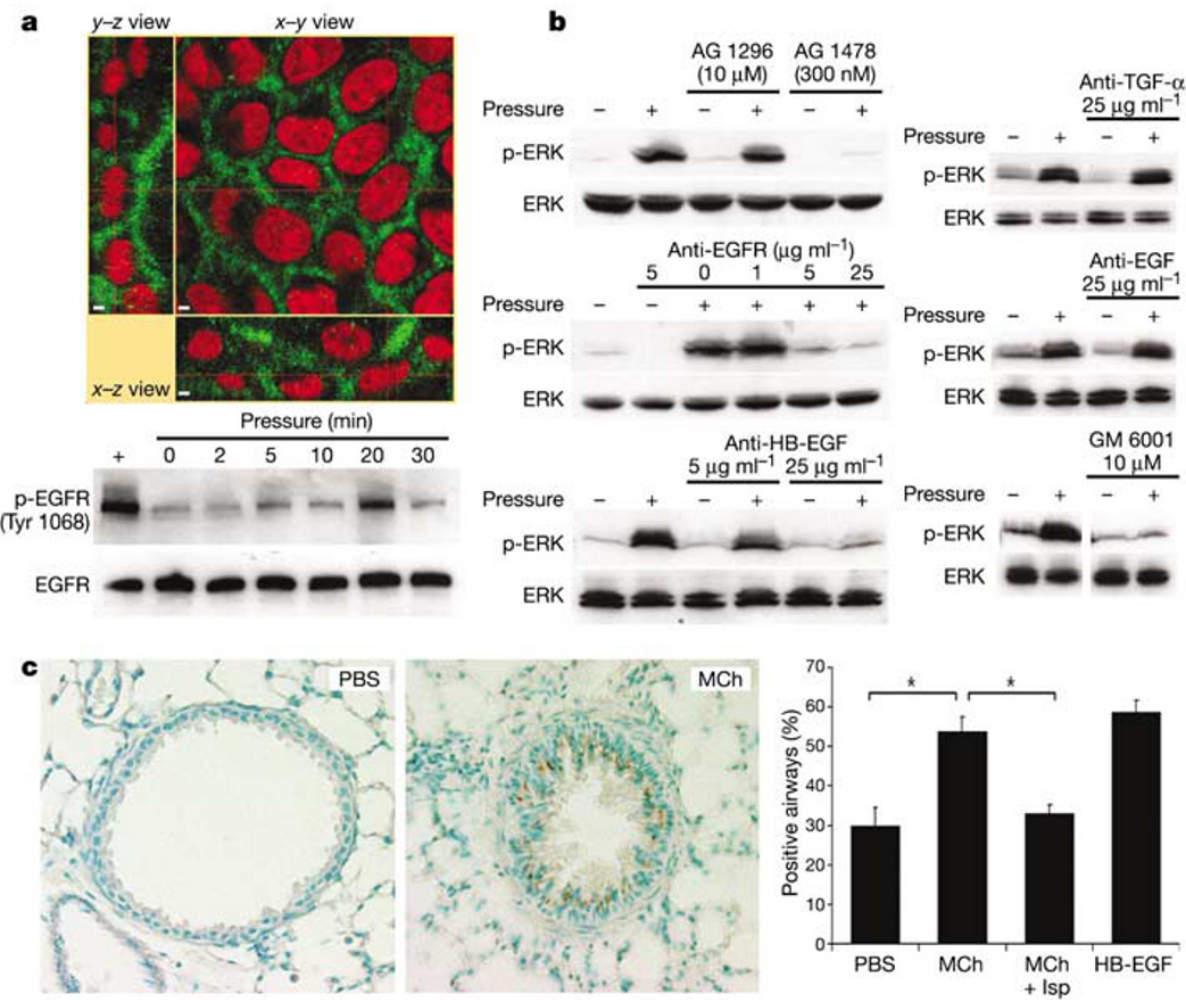

Figure 2 The EGFR mediates transduction of compressive stress. a, NHBE cells exhibit basolaterally polarized distribution of EGFR (green) lining the LIS; cell nuclei are counterstained with propidium iodide (red). Scale bars indicate $2 \mu \mathrm{m}$. The EGFR is rapidly phosphorylated (Tyr 1068) in response to transcellular compressive stress (pressure). b, NHBE cells exhibit ERK phosphorylation after 30 min exposure to transcellular pressure (20-30 $\mathrm{cm} \mathrm{H}_{2} \mathrm{O}$ ); this response is attenuated in the presence of the EGFR inhibitor AG 1478 but not the platelet-derived growth factor receptor inhibitor AG 1296, and is also attenuated by a function-blocking antibody that prevents ligand binding to the EGFR, a neutralizing antibody against HB-EGF, and the matrix metalloprotease inhibitor GM 6001, but not neutralizing antibodies against EGF and TGF- $\alpha$. All blots are representative of at least two independent experiments. c, Representative images show the increased immunostaining (brown) for the phosphorylated EGFR (Tyr 1068) in mouse lungs perfused for 5 min with MCh ( $1 \mathrm{mM}$ ) relative to those perfused with PBS. The increased staining (percentage positive airways; asterisk $=P<0.0005$, one-factorial analysis of variance and Bonferroni/Dunn test, $n=4-5$, mean \pm s.e.m.) was reversed by pre-treatment with isoproterenol (Mch + Isp), which abrogated the airway constriction. Perfusion with $10 \mathrm{ng} \mathrm{ml}^{-1} \mathrm{HB}-\mathrm{EGF}$ is included as a positive control. 
might result from EGFR transactivation secondary to G-proteincoupled receptor activation ${ }^{6,11,24}$ or ATP release ${ }^{8,25,26}$. Pre-treatment with either pertussis toxin or the extracellular ATP scavenger apyrase was incapable of attenuating the ERK phosphorylation response to compression (Supplementary Fig. 1).

We next considered whether it is feasible that the geometric alterations in the LIS are sufficient to stimulate EGFR signalling by modulating ligand availability and receptor occupancy ${ }^{27-29}$. EGF family ligands that are shed into LIS undergo molecular diffusion near the cell surface until either they are bound by cell-surface receptors, or they escape into the basal medium ${ }^{27}$. To describe the steady-state concentration profile of ligand molecules in the LIS, and the dependence of this concentration profile on LIS geometry, we solved the mass balance equation for ligand shedding and diffusion, leading to the expression $c=c_{\mathrm{m}}+\left(h^{2}-X^{2}\right) r /(D w)$. The concentration $c$ in the LIS exceeds the concentration $c_{\mathrm{m}}$ in the medium, or in the underlying tissue in vivo, owing to the autocrine ligand release rate $r$. The epithelium allows outflux of the released ligand to the medium through the basal LIS opening but not across the tight junctions at the apical surface of the cells, and so the concentration depends on the distance $X$ measured from the apex of the LIS (Fig. 3a). Assuming a uniform distribution of ligand shedding, constant in time, the concentration of ligands is highest adjacent to the tight junctions and decreases quadratically, reaching $c_{\mathrm{m}}$ at the basal LIS opening, where $X=h$, the LIS height. The difference in ligand concentration from that in the basal medium is proportional to the shedding rate $r$ and inversely proportional to the diffusion constant of the ligand, $D$. Notably, we find that the ligand concentration is inversely proportional to the LIS width $w$. Thus, our analysis predicts that the effective ligand concentration to which cells are exposed is strongly dependent on the geometry of the LIS, which is itself a function of the mechanical environment of the

a
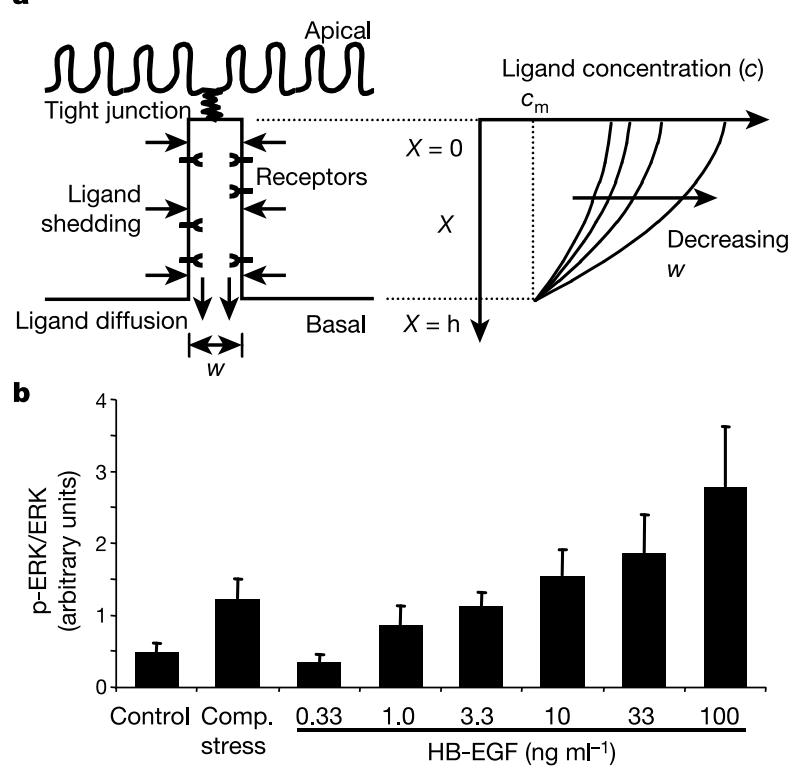

Figure 3 LIS width regulates autocrine signalling. a, Schematic of the epithelial LIS showing the boundary conditions regulating EGF family ligand diffusion, and example profiles of ligand concentration within the LIS. b, ERK phosphorylation elicited by compressive stress compared to 3.3-fold increasing concentrations of HB-EGF (mean \pm s.e.m., $n=5$ ). Concentrations of HB-EGF $<0.33 \mathrm{ng} \mathrm{ml}^{-1}$ had no effect on ERK phosphorylation. We thus assume that the baseline local concentration of EGF family ligands in the LIS is between 0.33 and $1.0 \mathrm{ng} \mathrm{ml}^{-1}$. From equation (7) we predict that the result of compressive stress should be most comparable to the effect of adding exogenous $\mathrm{HB}-\mathrm{EGF}$ to increase this concentration approximately tenfold, to between 3.3 and $10 \mathrm{ng} \mathrm{ml}^{-1}$. tissue. On the basis of this analysis we directly compared compressive stress to stimulation with soluble HB-EGF. In our imaging studies we measured an $87 \pm 10 \%$ decrease in LIS volume, which should yield an approximately eightfold increase in HB-EGF concentration. Compressive stress produced a change in ERK phosphorylation equivalent to a roughly tenfold increase in HB-EGF concentration (Fig. 3b), consistent with our prediction.

Our findings support the hypothesis that cells can sense mechanical stress through autocrine loops localized to compliant extracellular spaces; this mechanism seems to be capable of acting both independently of other known mechanosensitive responses and in concert with other mechanisms to broaden and amplify the response. Spatially limited autocrine loops occur in a wide variety of cell types, especially in epithelia where they are frequently localized in the LIS. Our results emphasize the high relative compliance of the LIS, and indicate that cells respond to local changes in ligand availability as a result of geometric alterations in their intercellular spaces. A unique aspect of this mechanochemical transduction mechanism is that it requires no direct conformational or metabolic alteration in any protein by mechanical stress. We speculate that such a transduction system could be tuned to perceive a wide range of deformations and stresses, and may be important in the sensation of and responses to physical forces in a number of biological systems.

\section{Methods}

\section{Cell culture}

NHBE cells (Clonetics-BioWhittaker) were cultured as previously described on uncoated microporous polyester inserts (Transwell-Clear, $0.4-\mu \mathrm{m}$ pore size, Costar) ${ }^{22}$. Experiments were carried out in minimal medium with no serum or growth factors except insulin $\left(5.7 \mu \mathrm{g} \mathrm{ml}^{-1}\right)$ and transferrin $\left(5 \mu \mathrm{g} \mathrm{ml}^{-1}\right)$. Function-blocking antibodies (all from R\&D Systems except anti-EGFR clone LA1; Upstate Biotechnology), GM 6001, AG 1478, AG 1296 (all from Calbiochem), and soluble HB-EGF (R\&D Systems) were applied to the basal surface of cultures only.

\section{Two-photon imaging}

NHBE cells were stained apically and basally with the fluorescent probes calcein AM $(10 \mu \mathrm{M})$, Celltracker green $(10 \mu \mathrm{M})$, or $70 \mathrm{kDa}$ fluorescein-dextran $(100 \mu \mathrm{M}$, all from Molecular Probes) diluted in culture medium. After incubation for $30 \mathrm{~min}$ each tissue culture insert was mounted on a custom-built two-photon microscope, and a silicon plug was used to create a pressure chamber over the apical surface of the cells. After a complete sequence of images was obtained under control conditions, an apical-to-basal transcellular pressure difference of $20 \mathrm{~cm} \mathrm{H}_{2} \mathrm{O}$ was applied. Imaging was then repeated within the same region of cells. Images were acquired at intervals of $0.5 \mu \mathrm{m}$ in the $z$ direction, with each image acquired at $256 \times 256$ pixels, $0.28 \mu \mathrm{m}$ pixel dimension. Image stacks were analysed using Spyglass Slicer (Spyglass).

\section{Western blotting}

Cell lysates were separated in $10 \%$ precast polyacrylamide gels (BioRad) ${ }^{22}$. Proteins were transferred to polyvinylidene difluoride membranes (Immobilon-P, Millipore), blocked with $5 \%$ milk, and incubated overnight $\left(4^{\circ} \mathrm{C}\right)$ in primary antibodies against ERK, phospho-ERK, EGFR, or phospho-EGFR (Tyr 1068; all from Cell Signaling Technology). Bands were visualized with horseradish peroxidase-conjugated anti-rabbit IgG $(1: 2,000)$ and enhanced chemiluminescence.

\section{Immunofluorescence}

Cells were fixed with $3 \%$ paraformaldehyde and stained with a mouse monoclonal antibody to the extracellular domain of the human EGFR (clone LA1, Upstate Biotechnology) and a secondary goat anti-mouse IgG antibody conjugated to Alexa Fluor 488 (Molecular Probes). Cell nuclei were counterstained with propidium iodide. Three-dimensional stacks of confocal images were obtained using a Sarastro 2000 confocal laser-scanning microscope.

\section{Isolated lung perfusion}

Lungs of male BALB/C mice (age 8 weeks) were tracheally perfused following the method of ref. 30 with PBS at a flow rate of $20 \mathrm{ml} \mathrm{h}^{-1}$. Perfusate exited the lung via small holes (5 per lobe) poked in the pleura ( 27 gauge needle). The airway opening pressure $\left(P_{\text {ao }}\right)$ at the trachea was continuously measured as an index of airway constriction. Perfusion with $\mathrm{MCh}$ induced a transient, concentration-dependent increase in $P_{\mathrm{ao}}$, with a maximal response at $1 \mathrm{mM}\left(\Delta P_{\text {ao }}=13 \mathrm{~cm} \mathrm{H}_{2} \mathrm{O}\right.$ at $\left.5 \mathrm{~min}\right)$. Perfusion with $10^{-4} \mathrm{M}$ Isp $1 \mathrm{~min}$ before and continuous with $\mathrm{MCh}$ perfusion attenuated $\mathrm{MCh}$-induced bronchial constriction by $78 \%$. Perfusion with Isp alone had little effect on $P_{\text {ao }}$. Perfused lungs from each group ( $n=4-5)$ were fixed by perfusion at $5 \mathrm{~min}$ with $4 \%$ paraformaldehyde. 


\section{Immunohistochemistry}

Paraffin-embedded lung sections were incubated with a rabbit polyclonal antibody against phopho-EGF receptor (Tyr 1068, Cell Signalling Technology); immunopositive areas were visualized with an avidin-biotin method using biotinylated goat anti-rabbit IgG secondary antibody (Vector Laboratories), the chromagen diaminobenzidine and methyl green counterstaining. Irrelevant IgG antibodies substituted for the primary antiserum were the negative controls. All cross-sectioned airways in each section were counted, and airways with any staining in the epithelium were counted as phospho-EGFR positive.

\section{Mass balance analysis of the LIS}

We approximate the geometry of the LIS as a two-dimensional gap of width $w$ and height $h$ between two surfaces (Fig. 3a), and assume that the rate of ligand shedding $r$, width $w$, and diffusion coefficient $D$ are independent of position $X$ along the apical-basal axis. Transport by convection is ignored. At steady state the rate of ligand entering the LIS via shedding from the cells bounding the LIS will be exactly balanced by the ligand leaving the LIS by diffusion through the basal opening of the LIS into the bulk medium. If we examine the mass transfer in an infinitesimal slice of LIS of width $w$ and thickness $\mathrm{d} X$ at position $X$, the flux into the slice can be written as:

$$
\text { influx }=2 r \mathrm{~d} X
$$

where $r$ is the density of ligand shedding per unit length, $\mathrm{d} X$ is the slice thickness, and the factor 2 comes from the two surfaces bounding the LIS in our idealized geometry. This influx is balanced by the net outflux due to diffusion, which is proportional to the spatial derivative of the ligand concentration gradient:

$$
\text { outflux }=-\frac{\mathrm{d}}{\mathrm{d} X}\left[w D \frac{\mathrm{d} c}{\mathrm{~d} X}\right]
$$

where $c$ is the concentration averaged over $y$. Equating influx and outflux, assuming constant $r, w$ and $D$, and integrating from 0 to $X$, yields:

$$
2 r \int_{0}^{X} \mathrm{~d} X=-w D \int_{0}^{X} \frac{\mathrm{d}}{\mathrm{d} X}\left[\frac{\mathrm{d} c}{\mathrm{~d} X}\right]
$$

Our boundary conditions are zero flux through the impermeable tight junction bounding the apical end of the LIS $(X=0)$, and ligand concentration equilibrates with the bulk medium $\left(c_{\mathrm{m}}\right)$ at the basal opening of the LIS $(X=h)$ :

$$
\left.\frac{\mathrm{d} c}{\mathrm{~d} X}\right|_{X=0}=0
$$

and

$$
\left.c\right|_{X=h}=c_{\mathrm{m}}
$$

Integrating equation (3) and using the boundary condition at $X=0$ (equation (4)), the following relation is obtained:

$$
\frac{\mathrm{d} c}{\mathrm{~d} X}=-\frac{2 r X}{w D}
$$

Solving this equation for $c(X)$ and satisfying the second boundary condition (equation (5)) yields:

$$
c=c_{\mathrm{m}}+\frac{r}{w D}\left(h^{2}-X^{2}\right)
$$

Received 2 February; accepted 5 April 2004; doi:10.1038/nature02543.

Published online 21 April 2004.

1. Blount, P. Molecular mechanisms of mechanosensation: big lessons from small cells. Neuron 37, 731-734 (2003).

2. Torday, J. S. \& Rehan, V. K. Mechanotransduction determines the structure and function of lung and bone: a theoretical model for the pathophysiology of chronic disease. Cell Biochem. Biophys. 37, 235-246 (2003).

3. Farge, E. Mechanical induction of Twist in the Drosophila foregut/stomodeal primordium. Curr. Biol. 13, 1365-1377 (2003)

4. Alenghat, F. J. \& Ingber, D. E. Mechanotransduction: all signals point to cytoskeleton, matrix, and integrins. Sci. STKE 2002, PE6 (2002).

5. Hamill, O. P. \& Martinac, B. Molecular basis of mechanotransduction in living cells. Physiol. Rev. 81, 685-740 (2001).

6. Gudi, S. R., Clark, C. B. \& Frangos, J. A. Fluid flow rapidly activates G proteins in human endothelia cells. Involvement of $\mathrm{G}$ proteins in mechanochemical signal transduction. Circ. Res. 79, 834-839 (1996).

7. Shyy, J. Y. \& Chien, S. Role of integrins in endothelial mechanosensing of shear stress. Circ. Res. 91, 769-775 (2002)

8. Dull, R. O., Tarbell, J. M. \& Davies, P. F. Mechanisms of flow-mediated signal transduction in endothelial cells: kinetics of ATP surface concentrations. J. Vasc. Res. 29, 410-419 (1992).

9. Ali, M. H. \& Schumacker, P. T. Endothelial responses to mechanical stress: where is the mechanosensor? Crit. Care Med. 30, S198-S206 (2002).

10. Han, O., Li, G. D., Sumpio, B. E. \& Basson, M. D. Strain induces Caco-2 intestinal epithelial proliferation and differentiation via PKC and tyrosine kinase signals. Am. J. Physiol. 275, G534-G541 (1998).

11. Correa-Meyer, E., Pesce, L., Guerrero, C. \& Sznajder, J. I. Cyclic stretch activates ERK1/2 via G proteins and EGFR in alveolar epithelial cells. Am. J. Physiol. Lung Cell. Mol. Physiol. 282, L883-L891 (2002).

12. Ressler, B., Lee, R. T., Randell, S. H., Drazen, J. M. \& Kamm, R. D. Molecular responses of rat trachea epithelial cells to transmembrane pressure. Am. J. Physiol. Lung Cell. Mol. Physiol. 278, L1264-L1272 (2000).

13. Lehoux, S. \& Tedgui, A. Signal transduction of mechanical stresses in the vascular wall. Hypertension 32, 338-345 (1998).

14. Wiggs, B. R., Hrousis, C. A., Drazen, J. M. \& Kamm, R. D. On the mechanism of mucosal folding in normal and asthmatic airways. J. Appl. Physiol. 83, 1814-1821 (1997).
15. Tschumperlin, D. J., Shively, J. D., Kikuchi, T. \& Drazen, J. M. Mechanical stress triggers selective release of fibrotic mediators from bronchial epithelium. Am. J. Respir. Cell Mol. Biol. 28, 142-149 (2003).

16. Swartz, M. A., Tschumperlin, D. J., Kamm, R. D. \& Drazen, J. M. Mechanical stress is communicated between different cell types to elicit matrix remodeling. Proc. Natl Acad. Sci. USA 98, 6180-6185 (2001).

17. Spring, K. R. \& Hope, A. Size and shape of the lateral intercellular spaces in a living epithelium. Science 200, 54-58 (1978).

18. Ohashi, Y., Motojima, S., Fukuda, T. \& Makino, S. Airway hyperresponsiveness, increased intracellular spaces of bronchial epithelium, and increased infiltration of eosinophils and lymphocytes in bronchial mucosa in asthma. Am. Rev. Respir. Dis. 145, 1469-1476 (1992).

19. Kondo, M., Finkbeiner, W. E. \& Widdicombe, J. H. Changes in permeability of dog tracheal epithelium in response to hydrostatic pressure. Am. J. Physiol. 262, L176-L182 (1992).

20. Polosa, R. et al. Expression of c-erbB receptors and ligands in human bronchial mucosa. Am. J. Respir. Cell Mol. Biol. 20, 914-923 (1999).

21. Vermeer, P. D. et al. Segregation of receptor and ligand regulates activation of epithelial growth factor receptor. Nature 422, 322-326 (2003).

22. Tschumperlin, D. J. et al. Bronchial epithelial compression regulates MAP kinase signaling and HBEGF-like growth factor expression. Am. J. Physiol. Lung Cell. Mol. Physiol. 282, L904-L911 (2002)

23. Dong, J. et al. Metalloprotease-mediated ligand release regulates autocrine signaling through the epidermal growth factor receptor. Proc. Natl Acad. Sci. USA 96, 6235-6240 (1999).

24. Prenzel, N. et al. EGF receptor transactivation by G-protein-coupled receptors requires metalloproteinase cleavage of proHB-EGF. Nature 402, 884-888 (1999).

25. Homolya, L., Steinberg, T. H. \& Boucher, R. C. Cell to cell communication in response to mechanical stress via bilateral release of ATP and UTP in polarized epithelia. J. Cell Biol. 150, 1349-1360 (2000).

26. Yamamoto, K. et al. Endogenously released ATP mediates shear stress-induced $\mathrm{Ca}^{2+}$ influx into pulmonary artery endothelial cells. Am. J. Physiol. Heart Circ. Physiol. 285, H793-H803 (2003).

27. Shvartsman, S. Y., Wiley, H. S., Deen, W. M. \& Lauffenburger, D. A. Spatial range of autocrine signaling: modeling and computational analysis. Biophys. J. 81, 1854-1867 (2001).

28. Lauffenburger, D. A., Oehrtman, G. T., Walker, L. \& Wiley, H. S. Real-time quantitative measurement of autocrine ligand binding indicates that autocrine loops are spatially localized. Proc. Natl Acad. Sci. USA 95, 15368-15373 (1998).

29. Shvartsman, S. Y. et al. Autocrine loops with positive feedback enable context-dependent cell signaling. Am. J. Physiol. Cell Physiol. 282, C545-C559 (2002).

30. Martins, M. A., Shore, S. A., Gerard, N. P., Gerard, C. \& Drazen, J. M. Peptidase modulation of the pulmonary effects of tachykinins in tracheal superfused guinea pig lungs. J. Clin. Invest. 85, 170-176 (1990).

Supplementary Information accompanies the paper on www.nature.com/nature.

Acknowledgements We thank J. Shively and J. Foley for technical assistance, G. Horsley for early work on EGFR signalling, and J. Lai for performing conventional confocal imaging. This work was supported by funding from the NHLBI (J.M.D. and R.D.K.), NIGMS (D.A.L.) and a Parker B. Francis Fellowship in Pulmonary Research (D.J.T.).

Competing interests statement The authors declare that they have no competing financial interests.

Correspondence and requests for materials should be addressed to J.M.D. (jdrazen@nejm.org).

\section{The ubiquitin ligase COP1 is a critical} negative regulator of p53

\section{David Dornan ${ }^{1}$, Ingrid Wertz ${ }^{1,5}$, Harumi Shimizu ${ }^{1}$, David Arnott ${ }^{2}$, Gretchen D. Frantz ${ }^{3}$, Patrick Dowd ${ }^{4}$, Karen 0' Rourke ${ }^{1}$, Hartmut Koeppen ${ }^{3}$ \& Vishva M. Dixit ${ }^{1}$}

\section{${ }^{1}$ Department of Molecular Oncology, ${ }^{2}$ Department of Protein Chemistry,} ${ }^{3}$ Department of Pathology and ${ }^{4}$ Department of Molecular Biology, Genentech, Inc., 1 DNA Way, South San Francisco, California 94080, USA

${ }^{5}$ Department of Human Physiology, University of California, Davis, California 95616, USA

COP1 (constitutively photomorphogenic 1) is a RING-fingercontaining protein that functions to repress plant photomorphogenesis, the light-mediated programme of plant development. Mutants of COP1 are constitutively photomorphogenic, and this has been attributed to their inability to negatively regulate the proteins LAF1 (ref. 1) and HY5 (ref. 2). The role of COP1 in mammalian cells is less well characterized ${ }^{3}$. Here we identify the tumour-suppressor protein p53 as a COP1-interacting protein. COP1 increases p53 turnover by targeting it for 